\title{
Establishment of a Model for Predicting the Recurrence of Recurrent Acute Pancreatitis in A Short Period of Time
}

Bo Yang ( $\sim$ yblyl9527@126.com )

Dazhou Central Hospital https://orcid.org/0000-0002-8598-6782

Peiyun Li

Dazhou Central Hospital

Honglan Liu

Dazhou Central Hospital

Xuesong Bai

Dazhou Central Hospital

Yijun Yu

Dazhou Central Hospital

Research article

Keywords: Model, recurrence, recurrent acute pancreatitis, short period

Posted Date: August 3rd, 2020

DOl: https://doi.org/10.21203/rs.3.rs-45624/v1

License: (c) (i) This work is licensed under a Creative Commons Attribution 4.0 International License. Read Full License 


\section{Abstract}

Background Little is known about what factors affect recurrence of recurrent acute pancreatitis (RAP) in a short-term. We performed a retrospective study of patients with RAP, and used non-invasive and quantitative biomarkers to establish a predictive model, so as to provide a basis for effectively predicting and preventing recurrence of RAP.

\section{Methods}

We performed a retrospective study of 261 RAP patients admitted to Dazhou Central Hospital from January 2008 through December 2018. The short-term group $(n=138)$ and the long-term group $(n=123)$ were compared. Eight clinical patient characteristics and past medical history were collected from admission records. The prediction model was developed by independent risk factors $\llbracket a n d$ the model to predict the effects were confirmed by the area under the ROC cure $\mathbb{Z}$

\section{Results}

The study found that $\beta 2$-microglobulin ( $\beta 2-\mathrm{MG})(\mathrm{OR}, 0.534 ; 95 \% \mathrm{Cl}, 0.329$ to 0.866$)$ and $\mathrm{C}$-reactive protein (CRP) $(\mathrm{OR}, 1.01 ; 95 \% \mathrm{Cl}, 1.002$ to 1.017$)$ were the independent predictors of short-term recurrence. Based on these Independent risk factors $₫$ a predicted model for short-term recurrence in RAP patients was

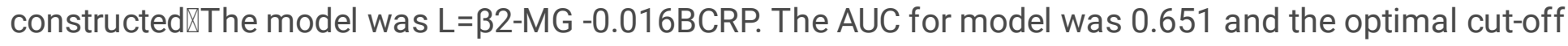
value was 0.7414 , with a sensitivity of $65.9 \%$ and a specificity of $63.4 \%$.

\section{Conclusions}

A new biomarker-based model for predicting risk of short-term recurrence in patients with RAP was satisfactorily developed. The model utilizing $\beta 2-M G$ and CRP may assist physicians in patient education and decision-making when preventing from recurrent attacks with RAP.

\section{Background}

Recurrent acute pancreatitis(RAP)is a clinical condition characterized by experienced 2 or more episodes of documented acute pancreatitis, separated by at least 3 months[1],which usually occurs in the setting of normal morphofunctional gland with self-limited edematous changes. Clinical data from all over the world show that the recurrence rate of acute pancreatitis is $10-30 \%[2-6]$. In addition to increasing the social burden and affecting the quality of life of the patient with RAP, current epidemiological and imaging data suggest that RAP has a higher risk of developing chronic pancreatitis(CP), and the more times of recurrence, the higher the risk of progression to $\mathrm{CP}[7,8]$. However, most of the literatures around the world focus on the analysis of high risk factors and imaging changes of RAP $[9,10]$ 区it has not been reported in the literature that there are high risk factors and predictive models related to short-term recurrence of RAP. Therefore, in this study, the clinical data of 261 patients with RAP were retrospectively analyzed to explore the clinical characteristics and risk factors of short-term recurrence of RAP, and to use 
of non-invasive and quantitative biomarkers to establish a predictive model, so as to provide a basis for effectively predicting and preventing of RAP recurrence.

\section{Methods}

\section{Patients}

A total of 261 RAP patients' written and electronic medical records who were admitted to Dazhou Central Hospital between January 2008 and December 2018 were assessed in this study. This retrospective study was approved by the ethics committee of Dazhou Central Hospital. Clinical data were retrieved and collected for retrospective analysis from the medical records of Dazhou Central Hospital database electronically. RAP was described as AP occurring at least 3 months after the previous episode. The number of prior attacks was determined based on the admitting history and physical note and past medical records. Patients with the following characteristics are excluded: (1) Insufficient diagnostic evidence; (2) Patients under 18; (3) Diagnosis of tumor or ongoing chemoradiotherapy; (4) Diagnosis of chronic pancreatitis; (5) Acute pancreatitis recurred within three months; (6) Case data are incomplete; (7) Chronic renal insufficiency and multiple organ failure; (8) Abdominal trauma or surgery.

\section{Data collection}

Eight clinical patient characteristics and past medical history, including gender, age, drinking and smoking status, gallstone status, length of hospital stay (indays), recurrence-free interval, diabetes, and hyperlipemia were collected from admission records. We obtained biochemical data at diagnosis and 12 hours after presentation.

\section{Statistical analysis}

Continuous variables are described as the means \pm standard deviations for normally distributed continuous variables. Median, 25th and 75th percentiles were calculated for non-normally distributed continuous variables. Numbers and percentages (\%) were used to describe categorical data. The characteristics of the study participants were analysed by an independent sample $t$-test or MannWhitney $\mathrm{U}$ test for continuous variables. A Chi-square test was used to analyze the association between categorical variables. Independent variables with $p<0.05$ in univariate analyses were included in a binary logistic regression model to select independent predictors of short-term recurrence. Results were presented as odds ratios (ORs) with $95 \%$ confidence intervals (Cls). Significant ( $p \otimes 0.05$ ) predictors were subsequently entered into the final multivariable prediction model. The area under the receiver operating characteristic (ROC) curve of the model to predict recurrence was calculated. The optimal cutoff value, sensitivity, and specificity were calculated on the basis of the ROC curve. All statistical analyses were performed using SPSS software, version 18.0 (SPSS Inc., Chicago, IL).

\section{Results}




\section{Patient characteristics}

Finally, a total of 261 patients were entered, consisted of 148 males and 113 females with a median recurrence-free interval of 11 months (interquartile range [IQR], $5 \mathrm{~m}-23.5 \mathrm{~m}$ ). All patients were divided into 2 groups according to interval of recurrence: the short-term group $(n=138)$ was defined as having an interval of recurrence less than the Median time, and the long-term group ( $n=123)$ greater than the Median time. Table 1 gives baseline characteristics of patient groups.

\section{Univariate and Multivariate Analysis}

Unvariable and multivariable analysis was conducted to investigate the prognostic factors in RAP patients underwent an interval of recurrence. Univariate analysis indicated that diabetes $(p<0.001)$, hyperlipemia $(p=0.005)$, drinking status $(p=0.018)$, Indays $(p=0.007)$, platelet distribution width (PDW) $(p$

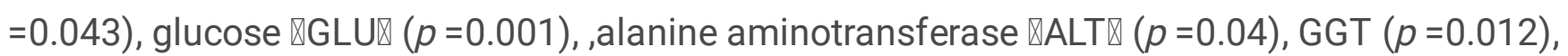
prealbumin $\triangle \mathrm{PA} \bigotimes(p=0.045), \beta 2-\mathrm{MG}(p=0.014)$ and CRP $(p=0.004)$ were suspected factors affecting the recurrence of RAP in a short time. (Table 1 ).

Multivariate analysis, including diabetes, hyperlipemia, achol, indays, PDW, GLU, ALT, GGT, PA, $\beta 2-M G$ and CRP, indicated that $\beta 2-M G$ and CRP were the independent predictor of short-term recurrence. (Table 2).

\section{Building independent predictive model.}

$B$ values of $\beta 2-M G$ and CRP ( $p \bowtie 0.05)$, derived from the binary logistic regression model, were constructed for the model function:

$L=\beta 2-M G-0.016 C R P$

ROC curve analysis was conducted and the area under the ROC curve (AUC) was used to assess the predictive accuracy of model function. (Figure 1)

Finally, the AUC for model was 0.651 and the optimal cut-off value was 0.7414 , with a sensitivity of $65.9 \%$ and a specificity of $63.4 \%$.

\section{Discussion}

RAP still represents a challenging disease, repeated episodes of which contribute substantially to the overall disease burden by prompting repeated hospitalization, adversely impacting quality of life as well as increasing the risk of progression to chronic pancreatitis and pancreatic tumor[2, 11-14]. RAP may run a milder clinical course than an index episode[15, 16], but the fatality rate of which was significantly higher than that of patients with first-episode AP[17]. Few reports have studied that the more frequent the onset of RAP, the more noticeable the decrease of the total pancreas volume and tail diameter, and the higher the incidence of $\mathrm{CP}[8,18]$. Therefore, the prediction of short-term recurrence of RAP is worthwhile to the early intervention and treatment of $\mathrm{CP}$. 
Although Some causes were a well-established risk factors for RAP[12, 18-21]. Theoretically, if the causes of acute pancreatitis existed, they could make acute pancreatitis to relapse repeatedly. In our retrospective study of patients with RAP, we found that diabetes, hyperlipemia, drinking status, ALT, GGT were involved in increased risk of RAP recurrence. Yadav et al showed that about 1/4 of patients with recurrent acute pancreatitis of known or unknown causes have underlying chronic pancreatic disease, which may invalidate treatment to remove known possible causes of acute pancreatitis [14]. Sajith et al found that clinical features and complications were the same in the idiopathic group and those with known etiology[22]. So, in the present study, we only found that $\beta 2-M G$ and CRP were independent risk factors for short-term recurrence of RAP.

Although challenging to prove in humans, the changes in the pancreas linked to increased AP susceptibility likely involve epigenetic changes, microanatomic changes, and/or immunological changes such as populating the parenchyma widespread with tissue histiocytes (eg, macrophages, dendritic cell) [23], excessive inflammatory reaction of the body[24], and changes of inflammatory mediators and cytokines[25].Thus, AP results in persistent changes that strongly predisposes to RAP. $\beta 2-M G$ is a low molecular weight protein that is synthesized by all human nucleated cells and platelets; It is indirectly involved in the regulation of the immune system response[26]. Some authors have found that serum $\beta 2-$ MG levels are elevated in patients with chronic pancreatitis[26-28],RAP is the most important risk factor for progression to end-stage $\mathrm{CP}[7,14]$. Recurrent episodes of acute pancreatitis may complicate the course of chronic subclinical pancreatitis, meaning they are the clinical expression of chronic pancreatitis diagnosed in an early phase, or otherwise they may themselves induce chronic lesions as a consequence of repeated damage. Therefore, in our prediction model, the accuracy of RAP recurrence prediction is positively correlated with $\beta 2-M G$, why which is it negatively related to CRP? This may be associated with pancreatic fibrosis after repeating recurrence of RAP. There is further emerging evidence to suggest that pancreatic fibrosis reduces severity in acute-on-chronic pancreatitis[29]. As such, recurrent attacks of pancreatitis may lead to increasing burden of parenchymal fibrosis, which in turn may be protective against the inflammatory cascade. Another speculated explanation is that protective immunological mechanisms are up-regulated in recurrent pancreatitis that not activates in the initial bout.

However, as a retrospective study predicting the recurrence of RAP, there were several limitations to the present study. Firstly, the present study was a single-center study enrolling limited number of patients. Secondly, our data lack dynamic follow-up during hospitalization and after discharging. Thirdly, CRP level is closely associated with the time of inflammation, but the measurement of CRP in our trial is the result of patients within 12 hours after admission, but the interval between onset and measurement is uniform. It may affect the results, or it may be one reason why the specificity and sensitivity of our prediction model are low. Eventually, this study only used biochemical detection technique, which may cause bias in the results. Further investigation using a multi-center and prospective study and involving radiomics technique is necessary in order to improve the accuracy of the results.

\section{Conclusion}


A new biomarker-based model for predicting risk of short-term recurrence in patients with RAP was satisfactorily developed. The model utilizing $\beta 2-M G$ and CRP may assist physicians in patient education and decision-making when preventing from recurrent attacks with RAP. Meanwhile, we still have to make more efforts to improve the specificity and sensitivity of our model.

\section{Abbreviations}

RAP, Recurrent acute pancreatitis; WBC, white blood cell; NEU, neutrophil; HGB, hemoglobin; PLT, platelet; PDW, platelet distribution width; GLU, glucose; ALT,alanine aminotransferase;AST,glutamic oxaloacetic transaminase;ALP,alkaline phosphatase;GGT,Y-glutamyl transferase;PA,prealbumin;CREA,creatinine;UA, uric acid;B2-MG, $\beta 2$-microglobulin;CYSC, cystatin $\mathrm{C} ; \mathrm{CHO}$, cholesterol;TG, triglyceride;CRP, C-reactive protein; $\mathrm{B}$, coefficient of regression; $\mathrm{SE}$, standard error; $\mathrm{df}$, free degree; $\mathrm{OR}$, odds ratio; $\mathrm{Cl}$, confidence interval; ROC, operator characteristic; AUC, area under curve.

\section{Declarations}

\section{Competing interests}

The authors declare that they have no competing interests.

\section{Funding}

None.

\section{Authors' contributions}

The study was designed by Bo Yang and Yijun Yu. Peiyun Li, Honglan Liu and Xuesong Bai collected clinical datas. Bo Yang carried out data analysis and wrote the paper.All authors read and approved the final manuscript.

\section{Acknowledgements}

None.

\section{References}

1. Guda NM, Muddana V, Whitcomb DC, Levy P, Garg P, Cote G, Uc A, Varadarajulu S, Vege SS, Chari ST: Recurrent acute pancreatitis: international state-of-the-science conference with recommendations. Pancreas 2018, 47(6):653-666.

2. Lankisch PG, Breuer N, Bruns A, Weber-Dany B, Lowenfels AB, Maisonneuve P. Natural history of acute pancreatitis: a long-term population-based study. Am J Gastroenterol. 2009;104(11):2797805; 
3. Vipperla K, Papachristou GI, Easler J, Muddana V, Slivka A, Whitcomb DC, Yadav D. Risk of and factors associated with readmission after a sentinel attack of acute pancreatitis. Clin Gastroenterol Hepatol. 2014;12(11):1911-9.

4. Whitlock TL, Repas K, Tignor A, Conwell D, Singh V, Banks PA, Wu BU. Early readmission in acute pancreatitis: incidence and risk factors. Am J Gastroenterol. 2010;105(11):2492.

5. Yan JG, Li YQ, Wang Q, Li SL, Li GQ, Ma J, Zeng XZ, Huang LY, Yuan SA, Liu CA. Analysis of the clinical features of recurrent acute pancreatitis in China. Journal of gastroenterology, 41(7):681685.

6. Gullo L, Migliori M, Pezzilli R, Oláh A, Farkas G, Levy P, Arvanitakis C, Lankisch P, Beger H. An update on recurrent acute pancreatitis: data from five European countries. American Journal of Gastroenterology, 97(8):1959-1962.

7. Sankaran SJ, Xiao AY, Wu LM, Windsor JA, Forsmark CE, Petrov MS. Frequency of progression from acute to chronic pancreatitis and risk factors: a meta-analysis. Gastroenterology. 2015;149(6):1490500. e1491.

8. DeSouza SV, Priya S, Cho J, Singh RG, Petrov MS. Pancreas shrinkage following recurrent acute pancreatitis: an MRI study. European radiology. 2019;29(7):3746-56.

9. Testoni PA. Acute recurrent pancreatitis: Etiopathogenesis, diagnosis and treatment. World $\mathrm{J}$ Gastroenterol. 2014;20(45):16891-901.

10. Chen Y, Chen TW, Wu CQ, Lin Q, Hu R, Xie CL, Zuo HD, Wu JL, Mu QW, Fu QS. Radiomics model of contrast-enhanced computed tomography for predicting the recurrence of acute pancreatitis. Eur Radiol. 2019;29(8):4408-17.

11. Takeyama Y. Recurrent acute pancreatitis: is it possible to exclude chronic pancreatitis? Journal of gastroenterology. 2006;41(7):722.

12. Kumar S, Ooi CY, Werlin S, Abu-El-Haija M, Barth B, Bellin MD, Durie PR, Fishman DS, Freedman SD, Gariepy C. Risk factors associated with pediatric acute recurrent and chronic pancreatitis: lessons from INSPPIRE. JAMA pediatrics. 2016;170(6):562-9.

13. Gurram KC, Czapla A, Thakkar S. Acute pancreatitis: pancreas divisum with ventral duct intraductal papillary mucinous neoplasms. Case Reports. 2014;2014:bcr2014205322.

14. Yadav D, O'connell M, Papachristou GI. Natural history following the first attack of acute pancreatitis. Am J Gastroenterol. 2012;107(7):1096-103.

15. Ranson JH, Pasternack BS. Statistical methods for quantifying the severity of clinical acute pancreatitis. J Surg Res. 1977;22(2):79-91.

16. Gullo L, Migliori M, Pezzilli R, Oláh A, Farkas G, Levy P, Arvanitakis C, Lankisch P, Beger H. An update on recurrent acute pancreatitis: data from five European countries. Am J Gastroenterol. 2002;97(8):1959-62.

17. Bertilsson S, Swärd P, Kalaitzakis E. Factors that affect disease progression after first attack of acute pancreatitis. Clin Gastroenterol Hepatol. 2015;13(9):1662-9. e1663. 
18. Magnusdottir BA, Baldursdottir MB, Kalaitzakis E, Björnsson ES. Risk factors for chronic and recurrent pancreatitis after first attack of acute pancreatitis. Scand J Gastroenterol. 2019;54(1):8794.

19. Pelli H, Sand J, Laippala P, Nordback I. Long-term follow-up after the first episode of acute alcoholic pancreatitis: time course and risk factors for recurrence. Scand J Gastroenterol. 2000;35(5):552-5.

20. Gloor B, Stahel PF, Müller CA, Worni M, Bûchler MW, Uhl W. Incidence and management of biliary pancreatitis in cholecystectomized patients: results of a 7-year study. J Gastrointest Surg. 2003;7(3):372-7.

21. Jackson WD. Pancreatitis: etiology, diagnosis, and management. Curr Opin Pediatr. 2001;13(5):44751.

22. Sajith KG, Chacko A, Dutta AK. Recurrent Acute Pancreatitis: Clinical Profile and an Approach to Diagnosis. Digestive Diseases \& Sciences, 55(12):3610-3616.

23. Whitcomb DC. Genetic risk factors for pancreatic disorders. Gastroenterology. 2013;144(6):1292302.

24. Szentkereszty Z, Kotan R, Kiss F, Klarik Z, Posan J, Furka I, Sapy P, Miko I, Peto K, Nemeth N. Effects of various drugs (flunixin, pentoxifylline, enoxaparin) modulating micro-rheological changes in cerulein-induced acute pancreatitis in the rat. Clin Hemorheol Microcirc. 2014;57(4):303-14.

25. Maléth J, Hegyi P. Ca2 + toxicity and mitochondrial damage in acute pancreatitis: translational overview. Philosophical Transactions of the Royal Society B: Biological Sciences. 2016;371(1700):20150425.

26. Pezzilli R, Billi P, Fiocchi M, Beltrandi E, Miglioli M. Serum beta 2-microglobulin in chronic diseases of the pancreas. Int J Pancreatol. 1995;17(2):161-6.

27. Rashid SA, Cooper EH, Axon AT, Eaves G. Serum beta 2-microglobulin in malignant and benign diseases of the stomach and pancreas. 1980, 33(4):112.

28. Hirsch-Marie H, Florent C, Levy VG. [Beta-2-microglobulin. Study in tumoral and nontumoral diseases of the pancreas]. Medecine chirurgie digestives. 1981;10(7):607-10.

29. Acharya C, Cline RA, Jaligama D, Noel P, Delany JP, Bae K, Furlan A, Baty CJ, Karlsson JM, Rosario BL. Fibrosis reduces severity of acute-on-chronic pancreatitis in humans. Gastroenterology. 2013;145(2):466-75.

\section{Tables}

Table 1. Single factor analysis of influencing factors between the short-term group and the long-term group. 


\begin{tabular}{|c|c|c|c|c|c|}
\hline variable & category & $\begin{array}{l}\text { short-term group } \\
\square n=138 \square\end{array}$ & $\begin{array}{l}\text { long-term group } \\
(n=123)\end{array}$ & $x^{2 / Z}$ & $p$ \\
\hline \multirow[t]{2}{*}{ Sex } & Male & 73(49.3\%) & $75(50.7 \%)$ & \multirow[t]{2}{*}{1.728} & \multirow[t]{2}{*}{0.189} \\
\hline & Female & $65(57.5 \%)$ & $48(42.5 \%)$ & & \\
\hline Age(year) & & $46(40 \sim 55)$ & 45(39 52) & -1.490 & 0.134 \\
\hline \multirow[t]{2}{*}{ Diabetes } & Yes & $57(70.4 \%)$ & $24(29.6 \%)$ & \multirow[t]{2}{*}{$14.43^{\star}$} & \multirow{2}{*}{0.001} \\
\hline & No & $81(45.0 \%)$ & $99(55.0 \%)$ & & \\
\hline \multirow[t]{2}{*}{ Hyperlipem } & Yes & $28 \bigotimes 73.7 \% \rrbracket$ & $10 \rrbracket 26.3 \% \rrbracket$ & \multirow[t]{2}{*}{$7.73^{\star}$} & \multirow[t]{2}{*}{0.005} \\
\hline & No & $110 \otimes 49.3 \% \rrbracket$ & $113 \rrbracket 50.7 \% \rrbracket$ & & \\
\hline \multirow[t]{3}{*}{ Cholelithiasis } & Yes & $7 \rrbracket 50.0 \% \rrbracket$ & $7 \rrbracket 50.0 \% \bigotimes$ & \multirow[t]{3}{*}{3.342} & \multirow[t]{3}{*}{0.188} \\
\hline & Removed & $75 \rrbracket 58.6 \% \rrbracket$ & $53 \llbracket 41.4 \% \rrbracket$ & & \\
\hline & No & $56 \rrbracket 47.1 \% \rrbracket$ & $63 \rrbracket 52.9 \% \rrbracket$ & & \\
\hline \multirow[t]{3}{*}{ Smoking } & Smoker & $54 \llbracket 48.7 \% \rrbracket$ & $57 \rrbracket 51.3 \% \rrbracket$ & \multirow[t]{3}{*}{1.677} & \multirow[t]{3}{*}{0.432} \\
\hline & $\begin{array}{l}\text { Former } \\
\text { smoker }\end{array}$ & $21 \rrbracket 60.0 \% \rrbracket$ & $14 \llbracket 40.0 \% \rrbracket$ & & \\
\hline & None & $63 \otimes 54.8 \% \bigotimes$ & $52 \llbracket 45.2 \% \rrbracket$ & & \\
\hline \multirow[t]{3}{*}{ Achol } & Drinker & $28 \rrbracket 39.4 \% \rrbracket$ & $43 \bowtie 60.6 \% \rrbracket$ & \multirow[t]{3}{*}{$7.993^{*}$} & \multirow[t]{3}{*}{0.018} \\
\hline & $\begin{array}{l}\text { Former } \\
\text { drinker }\end{array}$ & $49 \rrbracket 62.0 \% \rrbracket$ & $30 \rrbracket 38.0 \% \rrbracket$ & & \\
\hline & None & $61 \otimes 55.0 \% \bigotimes$ & $50 \rrbracket 45.0 \% \rrbracket$ & & \\
\hline Indays & & $9(7 \sim 13)$ & 11(8 17) & $-2.689 *$ & 0.007 \\
\hline 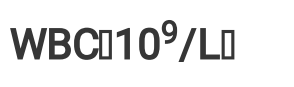 & & 11.97(9.37 15.57) & 12.86(9.53 16.54) & -1.291 & 0.197 \\
\hline 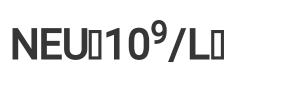 & & $9.8(7.54 \sim 13.68)$ & 10.86(7.54 14.33) & -1.221 & 0.222 \\
\hline HGB\#" $\mathrm{g} / \mathrm{L} \square$ & & $142.68 \pm 24.24$ & $148.11 \pm 23.51$ & 0.241 & 0.624 \\
\hline PLT010/L口 & & 132(101.50 169.75) & 136(101.00 166.00) & -0.242 & 0.809 \\
\hline PDW0\%] & & 18.4(17.00 20.64) & 17.8(17.00 18.80) & $-2.022^{*}$ & 0.043 \\
\hline GLUImmol/L】 & & $9.46(6.68 \sim 13.96)$ & 7.37(6.39 10.23) & $-3.204^{*}$ & 0.001 \\
\hline ALTDU/L口 & & 24.65(17.00 41.50) & $30.4(18.90 \sim 52.00)$ & $-2.053^{*}$ & 0.040 \\
\hline ASTLU/LD & & 24.4(18.73 40.40) & 27.2(20.20 45.00) & -1.924 & 0.054 \\
\hline ALPIU/L口 & & 80.4(62.90 105.03) & 76.3(62.10 100.00) & -0.671 & 0.502 \\
\hline
\end{tabular}




\begin{tabular}{|c|c|c|c|c|}
\hline GGTOU/L & 41.75(23.00 92.60) & $57 \rrbracket 29.00 \sim 141.00)$ & $-2.519 *$ & 0.012 \\
\hline PAImg/L口 & 171.95(134.58 238.50) & 187.6(150.2 260) & $-2.002^{\star}$ & 0.045 \\
\hline CREADumol/L] & 60.75(47.95 71.4) & 63(49.3 75.9) & -1.266 & 0.206 \\
\hline UADumol/Lם & 283(240.58 337.95) & $309.3(240 \sim 370)$ & -1.795 & 0.073 \\
\hline 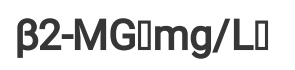 & $1.28(0.98 \sim 1.65)$ & $1.15(0.82 \sim 1.45)$ & $-2.468^{*}$ & 0.014 \\
\hline CYSCImg/L】 & $0.69(0.6 \sim 0.8)$ & $0.71(0.6 \sim 0.8)$ & -0.460 & 0.645 \\
\hline CHOLU/L】 & $5.17(3.97 \sim 7.08)$ & $5.08(4.25 \sim 6.71)$ & -0.078 & 0.938 \\
\hline TGImmol/L & $4.2(1.38 \sim 12)$ & 3.81(1.47 11.88) & -0.082 & 0.935 \\
\hline CRPImg/L口 & $14.1(5 \sim 56.6)$ & $45.5(5.92 \sim 85.38)$ & $-2.886^{*}$ & 0.004 \\
\hline
\end{tabular}

* $p \varangle 0.05$ was considered statistically significant. \# indicates the data distribution was tested for normality with the Shapiro-Wilk test.

Table 2. Multiple Logistic Regression Analysis for Risk Factors.

\begin{tabular}{|llllllll|}
\hline Variable & B & SE & Wald $\chi 2$ & df & $p$ & OR & $95 \% \mathrm{Cl}$ \\
\hline abetes(1) & -0.566 & 0.427 & 1.762 & 1 & 0.184 & 0.568 & $0.246-1.31$ \\
\hline Hyperlipem(1) & -0.807 & 0.435 & 3.446 & 1 & 0.063 & 0.446 & $0.19-1.046$ \\
\hline Achol & & & 2.241 & 2 & 0.326 & & \\
\hline Achol(1) & 0.154 & 0.346 & 0.199 & 1 & 0.655 & 1.167 & $0.593-2.298$ \\
\hline Achol(2) & -0.37 & 0.331 & 1.254 & 1 & 0.263 & 0.691 & $0.361-1.32$ \\
\hline Indays & 0.043 & 0.025 & 3.048 & 1 & 0.081 & 1.044 & $0.995-1.095$ \\
\hline PDW & -0.053 & 0.047 & 1.297 & 1 & 0.255 & 0.948 & $0.865-1.039$ \\
\hline GLU & -0.059 & 0.047 & 1.597 & 1 & 0.206 & 0.943 & $0.861-1.033$ \\
\hline ALT & 0 & 0.002 & 0.01 & 1 & 0.921 & 1 & $0.996-1.004$ \\
\hline GGT & 0.001 & 0.001 & 0.578 & 1 & 0.447 & 1.001 & $0.999-1.003$ \\
\hline PA & 0.001 & 0.001 & 0.456 & 1 & 0.499 & 1.001 & $0.998-1.004$ \\
\hline B2-MG & -0.627 & 0.247 & 6.454 & 1 & $0.011 *$ & 0.534 & $0.329-0.866$ \\
\hline CRP & 0.01 & 0.004 & 6.861 & 1 & $0.009 *$ & 1.01 & $1.002-1.017$ \\
\hline Constant & 1.42 & 1.106 & 1.649 & 1 & 0.199 & 4.138 & 4.138 \\
\hline
\end{tabular}

* $p \bigotimes 0.05$ was considered statistically significant. 
Figures

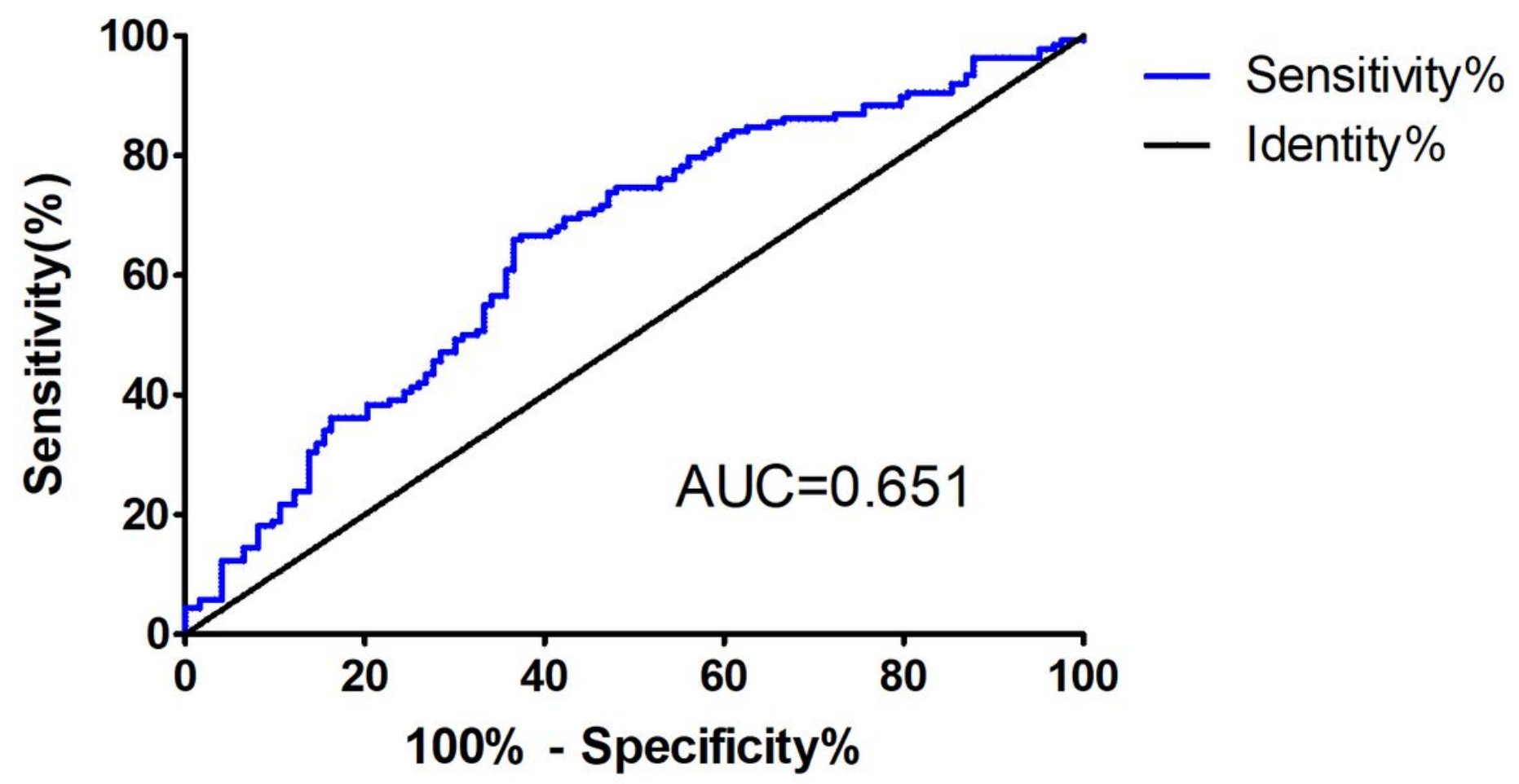

Figure 1

The ROC curve analyses of the predictive accuracy of model function. 